\section{Imaging features of complex sclerosing lesions of the breast}

\author{
Joo Hwa Myong ${ }^{1}$, Byung Gil Choi ${ }^{1}$, Sung Hun Kim¹, Bong Joo Kang ${ }^{1}$, Ahwon Lee², \\ Byung Joo Song ${ }^{3}$ \\ Departments of ${ }^{1}$ Radiology, ${ }^{2}$ Pathology, and ${ }^{3}$ Surgery, Seoul St. Mary's Hospital, The Catholic \\ University of Korea, Seoul, Korea
}

Purpose: The purpose of this study was to evaluate the imaging features of complex sclerosing lesions of the breast and to assess the rate of upgrade to breast cancer.

Methods: From March 2008 to May 2012, seven lesions were confirmed as complex sclerosing lesions by ultrasonography-guided core needle biopsy. Final results by either surgical excision or follow-up imaging studies were reviewed to assess the rate of upgrade to breast cancer. Two radiologists retrospectively analyzed the imaging findings according to the Breast Imaging Reporting and Data System classification.

Results: Five lesions underwent subsequent surgical excision and two of them revealed ductal carcinoma in situ $(n=1)$ and invasive ductal carcinoma $(n=1)$. Our study showed a breast cancer upgrade rate of $28.6 \%$ ( 2 of 7 lesions). Two lesions were stable on imaging follow-up beyond 1 year. The mammographic features included masses $(n=4,57.1 \%)$, architectural distortion $(n=2$, $28.6 \%)$, and focal asymmetry $(n=1,14.3 \%)$. Common B-mode ultrasonographic features were irregular shape $(n=6,85.7 \%)$, spiculated margin $(n=5,71.4 \%)$, and hypoechogenicity $(n=7$, $100 \%)$. The final assessment categories were category $4(n=6,85.7 \%)$ and category $5(n=1$, $14.3 \%)$.

Conclusion: The complex sclerosing lesions were commonly mass-like on mammography and showed the suspicious ultrasonographic features of category 4. Due to a high underestimation rate, all complex sclerosing lesions by core needle biopsy should be excised.

Keywords: Breast; Ultrasonography; Mammography; Breast diseases

\section{Introduction}

Complex sclerosing lesions are rare benign breast lesions that include sclerosing lesions with a variety of epithelial proliferative lesions [1]. Radial scars exceeding $1 \mathrm{~cm}$ in diameter are known to be complex sclerosing lesions [2-4]. Such radial scars are characterized by a central fibroelastic zone from which tubular structures radiate [5]. Complex sclerosing lesions have all of the features of radial scars and may exhibit nodular masses with or without other changes, such as papillomas, apocrine changes, and sclerosing adenosis around the periphery [5].

Most lesions are benign, but malignant cancer may coexist or develop [6]. Several studies have published data regarding radial scars and the radial scar/complex sclerosing lesion combination [7-

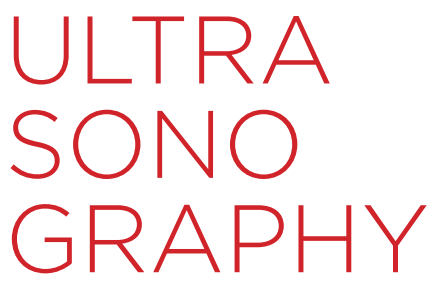

\section{ORIGINAL ARTICLE}

http://dx.doi.org/10.14366/usg.13015 pISSN: 2288-5919 • elSSN: 2288-5943 Ultrasonography 2014;33:58-64

Received: October 16, 2013

Revised: October 31, 2013

Accepted: November 27, 2013

Correspondence to:

Byung Gil Choi, MD, Department of Radiology, Seoul St. Mary's Hospital, The Catholic University of Korea, 222 Banpo-daero, Seocho-gu, Seoul 137701 , Korea

Tel. +82-2-2258-6242

Fax. +82-2-599-6771

E-mail: cbg@catholic.ac.kr

This is an Open Access article distributed under the terms of the Creative Commons Attribution NonCommercial License (http://creativecommons.org/ licenses/by-nc/3.0/) which permits unrestricted noncommercial use, distribution, and reproduction in any medium, provided the original work is properly cited.

Copyright @ 2014 Korean Society of Ultrasound in Medicine (KSUM)

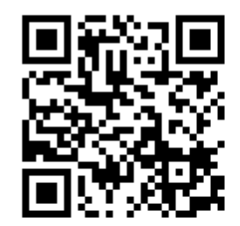

How to cite this article:

Myong JH, Choi BG, Kim SH, Kang BJ, Lee $A$, Song $B J$. Imaging features of complex sclerosing lesions of the breasts. Ultrasonography. 2014 Jan;33(1):58-64. 
9], but to date, few studies have reported imaging results of complex sclerosing lesions alone [9]. In particular, previous studies have rarely reported the radiological features of complex sclerosing lesions according to the Breast Imaging Reporting and Data System (BI-RADS) [9]. There are conflicting data regarding the risk of breast cancer subsequent to a complex sclerosing lesion [10-12]. The present study was conducted to assess the rate of upgrade from a complex sclerosing lesion to breast cancer and to evaluate the imaging features of complex sclerosing lesions according to BI-RADS [13].

\section{Materials and Methods}

This study was approved by our Institutional Review Board, which waived the requirement for patient consent for this retrospective review.

From March 2008 to May 2012, seven lesions were confirmed to be complex sclerosing lesions by ultrasonography-guided core needle biopsy. In all of the patients, subsequent surgical excision or a follow-up imaging study was performed and reviewed to assess the breast cancer upgrade rate. Medical records and pathology results were reviewed for the evaluation of clinical features and histology.

Mammography and ultrasonography were performed in all of the patients. Mammographic images were acquired using a Mammomat Inspiration (Siemens, Erlangen, Germany) and Selenia (Hologic, Bedford, MA, USA). The ultrasonographic images were acquired using a 7-15 MHz linear probe (HDI 5000, Advanced Technology Laboratories, Bothell, WA, USA; iU22 Ultrasound System, Philips Ultrasound, Bothell, WA, USA) and a 6-14 MHz linear probe (EUB8500 scanner, Hitachi Medical, Tokyo, Japan).

Two radiologists retrospectively analyzed the mammographic findings for shape, margin, and density, and the ultrasonographic findings for shape, margin, internal echogenicity, and posterior acoustic features including category according to the American College of Radiology BI-RADS [13].

\section{Results}

The clinical findings of the patients are summarized in Table 1. The median age of the patient was 34 years old (age range, 25 to 51 years). The lesions varied in size from 1.1 to $2.6 \mathrm{~cm}$ (median, $1.7 \mathrm{~cm}$ ). On physical examination, two patients complained of a palpable mass, and one complained of mastalgia. The remaining four patients were asymptomatic. Five lesions underwent subsequent surgical excision: complex sclerosing lesion $(n=2)$ (Fig. 1), complex sclerosing lesion with atypical ductal hyperplasia $(n=1)$ (Fig. 2), ductal carcinoma in situ ( $n=1$ ) (Fig. 3), and invasive ductal carcinoma $(n=1)$. Two lesions showed stable imaging findings on follow-up imaging studies (12 and 24 months).

The mammographic and ultrasonographic findings of the complex sclerosing lesions are summarized in Tables 2 and 3. The lesions appeared as isodense masses in $57.1 \%$ of the cases (4 of 7), with an oval $(50 \%, 2$ of 4$)$, lobular $(25 \%, 1$ of 4$)$, or irregular shape $(25 \%, 1$ of 4$)$. The margin was obscured $(50 \%, 2$ of 4$)$, spiculated $(25 \%, 1$ of 4$)$, or indistinct $(25 \%, 1$ of 4$)$. The lesions that appeared as isodense masses did not exhibit a central radiolucent area or calcifications (Fig. 2A). The others exhibited architectural distortion $(28.6 \%, 2$ of 7$)$ (Fig. 1A) or focal asymmetry (14.3\%, 1 of 7) (Fig. $3 \mathrm{~A})$ with a central radiolucent area.

All of the lesions appeared as hypoechoic masses on ultrasonography. The shapes were irregular $(85.7 \%, 6$ of 7$)$ or oval $(14.3 \%, 1$ of 7$)$. The margins were spiculated $(71.4 \%, 5$ of 7$)$, microlobulated $(14.3 \%, 1$ of 7$)$, or indistinct $(14.3 \%, 1$ of 7$)$. In the final assessment, the lesions were classified as BI-RADS category 4C with moderate concern regarding malignancy $(42.9 \%, 3$ of 7 ) (Figs. 1B, $3 \mathrm{~B})$, category $4 \mathrm{~B}$ with intermediate suspicion of malignancy $(28.6 \%$, 2 of 7$)$, and category $4 A$ with low suspicion of malignancy $(14.3 \%$, 1 of 7). Moreover, one lesion was classified as BI-RADS category 5, with characteristics highly suggestive of malignancy $(14.3 \%, 1$ of 7$)$ (Fig. 2B)

Upgraded lesions are those with an initial histopathologic

Table 1. Clinical and pathologic features of complex sclerosing lesions

\begin{tabular}{cccllc}
\hline Patient no. & Age $(\mathrm{yr})$ & Size $(\mathrm{cm})$ & Symptom & \multicolumn{1}{c}{ Histology by CNB } & Histology by surgery \\
\hline 1 & 34 & 1.1 & None & Complex sclerosing lesion & Complex sclerosing lesion \\
2 & 34 & 1.5 & Mastalgia & Complex sclerosing lesion & - \\
3 & 25 & 2.0 & None & Complex sclerosing lesion & Complex sclerosing lesion \\
4 & 33 & 2.6 & None & Complex sclerosing lesion & Complex sclerosing lesion with ADH \\
5 & 43 & 1.7 & Palpable mass & Complex sclerosing lesion with ADH & Invasive ductal carcinoma \\
6 & 50 & 2.0 & Palpable mass & Complex sclerosing lesion with atypical & proliferation disease \\
7 & 51 & 1.7 & None & Complex sclerosing lesion with ADH & Ductal carcinoma in situ \\
\hline
\end{tabular}

CNB, ultrasonography-guided core needle biopsy; ADH, atypical ductal hyperplasia. 
Table 2. Mammographic features of complex sclerosing lesions

\begin{tabular}{|c|c|c|c|c|c|}
\hline Patient no. & Abnormal finding & Shape & Margin & Density & Calcification \\
\hline 1 & Architectural distortion & - & - & - & No \\
\hline 2 & Mass & Oval & Obscured & Isodense & No \\
\hline 3 & Architectural distortion & - & - & - & No \\
\hline 4 & Mass & Irregular & Spiculated & Isodense & No \\
\hline 5 & Mass & Lobular & Obscured & Isodense & No \\
\hline 6 & Mass & Oval & Indistinct & Isodense & No \\
\hline 7 & Focal asymmetry & - & - & - & No \\
\hline
\end{tabular}

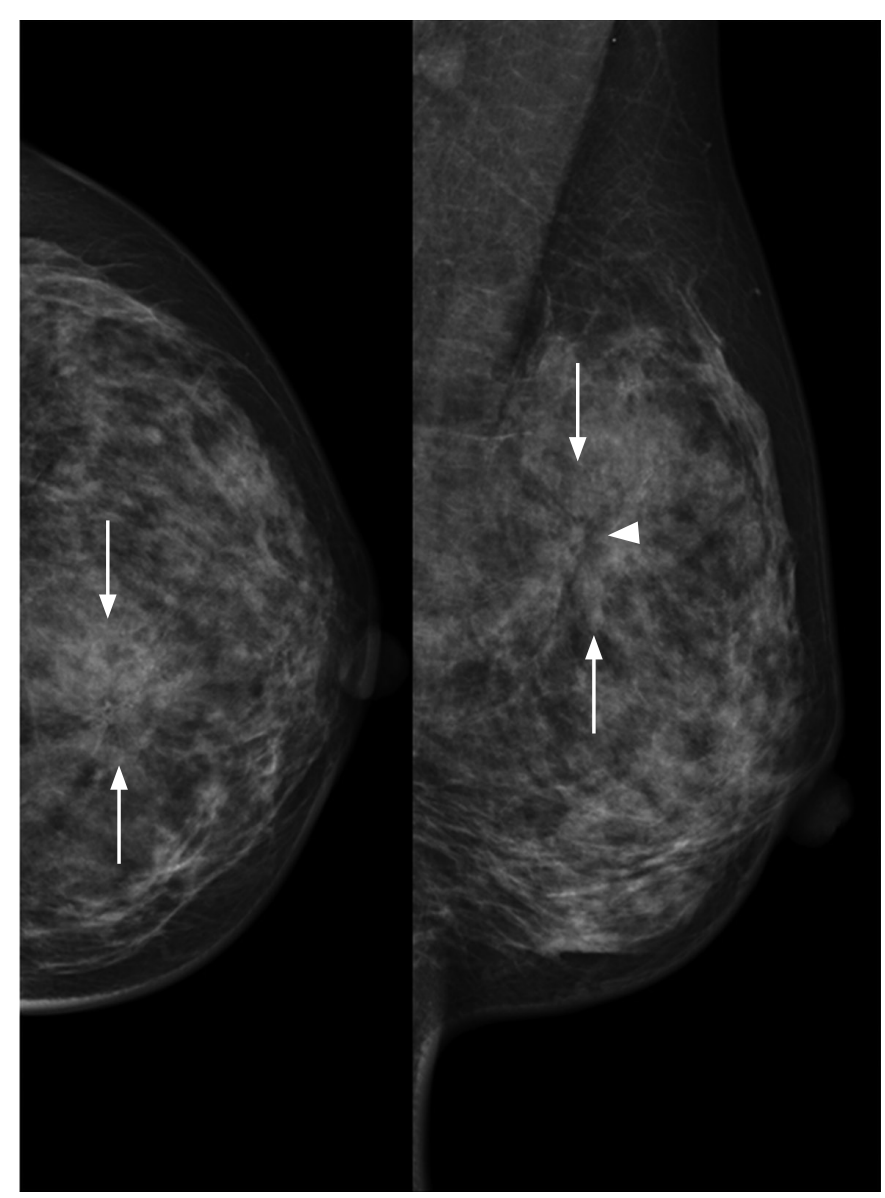

A

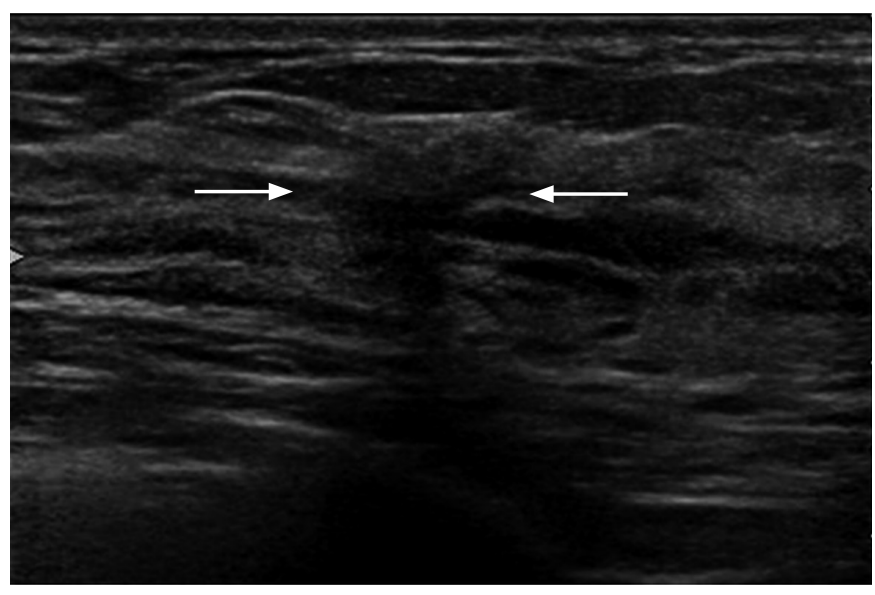

B

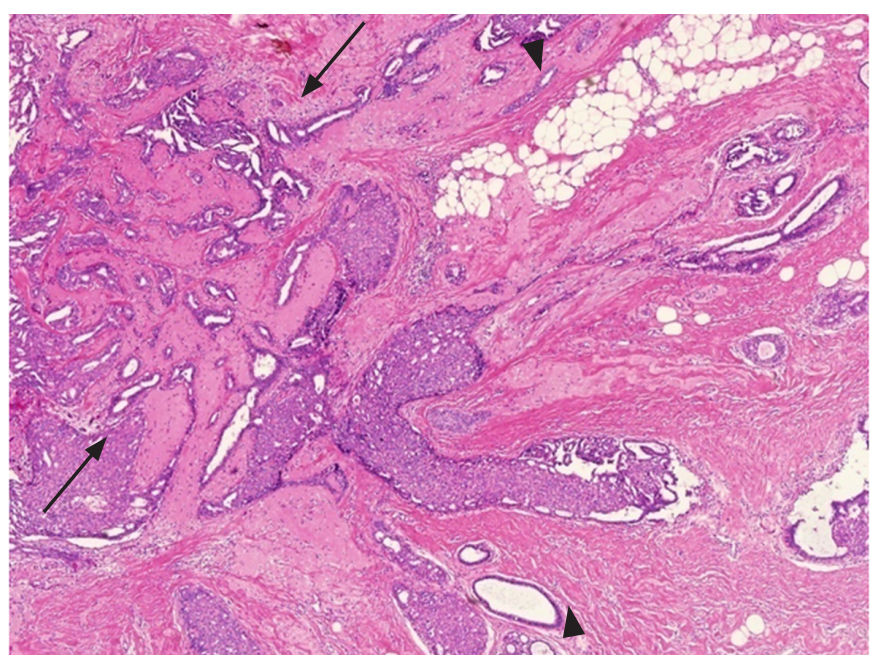

C

Fig. 1. Complex sclerosing lesion in a 34-year-old woman with no symptoms (patient 1).

A. A mammogram shows focal architectural distortion (arrows) with a central radiolucent area (arrowhead). B. An ultrasonogram reveals an irregular, non-parallel, spiculated hypoechoic mass (arrows). Upon BI-RADS assessment, it was classified as category 4C, with moderate suspicion of malignancy. C. A photomicrograph of a histologic specimen $(\mathrm{H \& E}, \times 40)$ reveals a central fibroelastic core surrounded by a radiating compressed ductal structure, compatible with complex sclerosing lesion (arrows and arrowheads). 
Table 3. Ultrasonographic features of complex sclerosing lesion

\begin{tabular}{|c|c|c|c|c|c|c|}
\hline Patient no. & Shape & Orientation & Margin & Internal echotexture & PAF & BI-RADS category \\
\hline 1 & Irregular & Non-parallel & Spiculated & Hypoechogenicity & No PAF & $4 C$ \\
\hline 2 & Oval & Parallel & Spiculated & Hypoechogenicity & No PAF & $4 \mathrm{~B}$ \\
\hline 3 & Irregular & Parallel & Spiculated & Hypoechogenicity & No PAF & $4 C$ \\
\hline 4 & Irregular & Parallel & Spiculated & Hypoechogenicity & Enhancement & 5 \\
\hline 5 & Irregular & Parallel & Indistinct & Hypoechogenicity & Enhancement & $4 \mathrm{~A}$ \\
\hline 6 & Irregular & Parallel & Microlobulated & Hypoechogenicity & Enhancement & $4 \mathrm{~B}$ \\
\hline 7 & Irregular & Parallel & Spiculated & Hypoechogenicity & No PAF & $4 C$ \\
\hline
\end{tabular}

PAF, posterior acoustic features; BI-RADS, Breast Imaging Reporting and Data System.

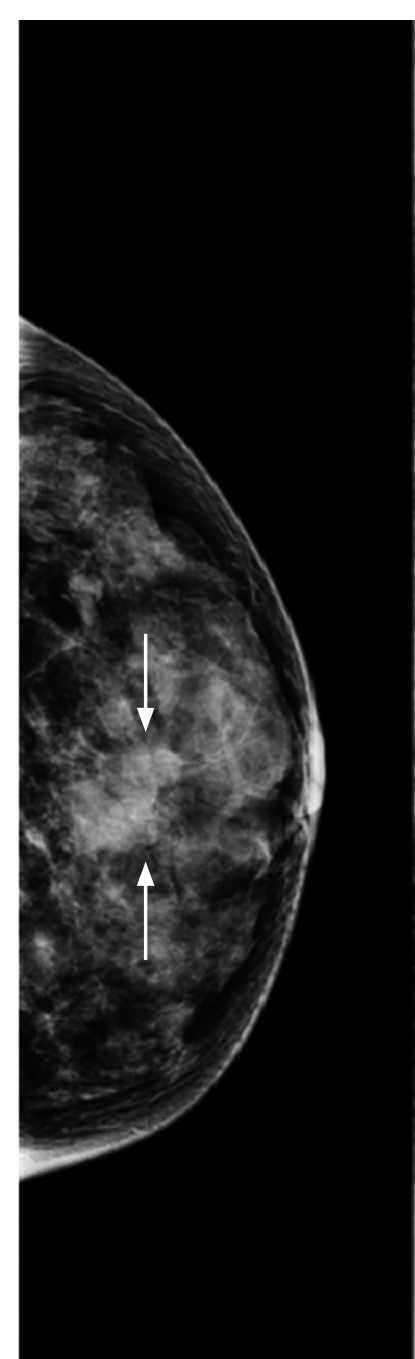

A

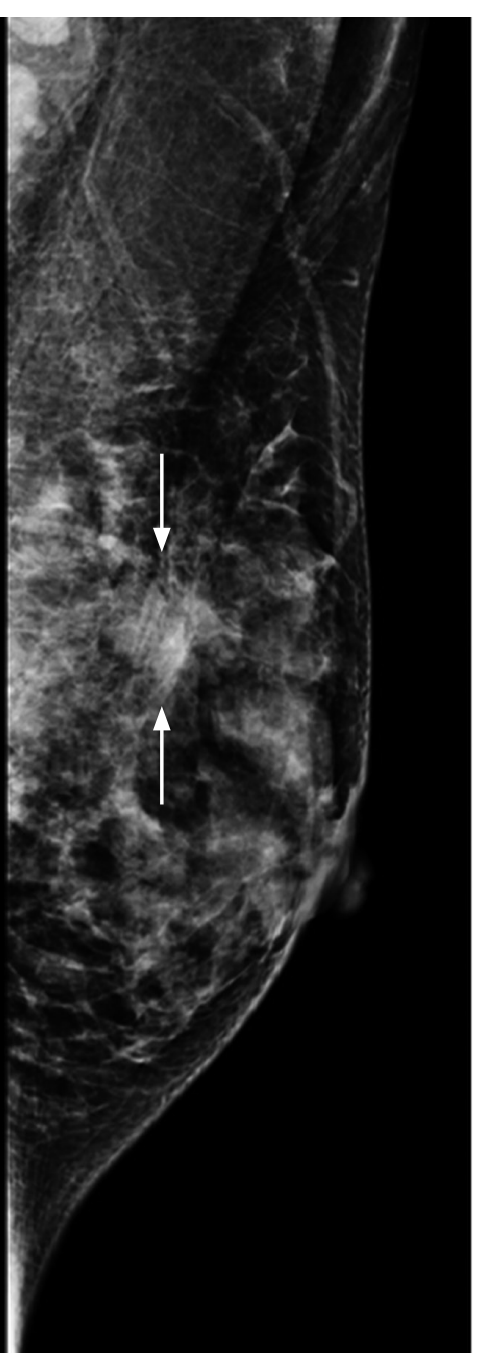

Fig. 2. A complex sclerosing lesion in a 33-year-old woman with no symptoms (patient 4).

A. A mammogram shows an irregular spiculated isodense mass (arrows) without calcification or a central radiolucent area. B. An ultrasonogram reveals an irregular, spiculated hypoechoic mass (arrows) with posterior acoustic enhancement (arrowheads). Upon BI-RADS assessment, it was classified as category 5 , highly suggestive of malignancy. C. A photomicrograph of a histologic specimen $(H \& E, \times 40)$ from excision reveals a complex sclerosing lesion (arrows).

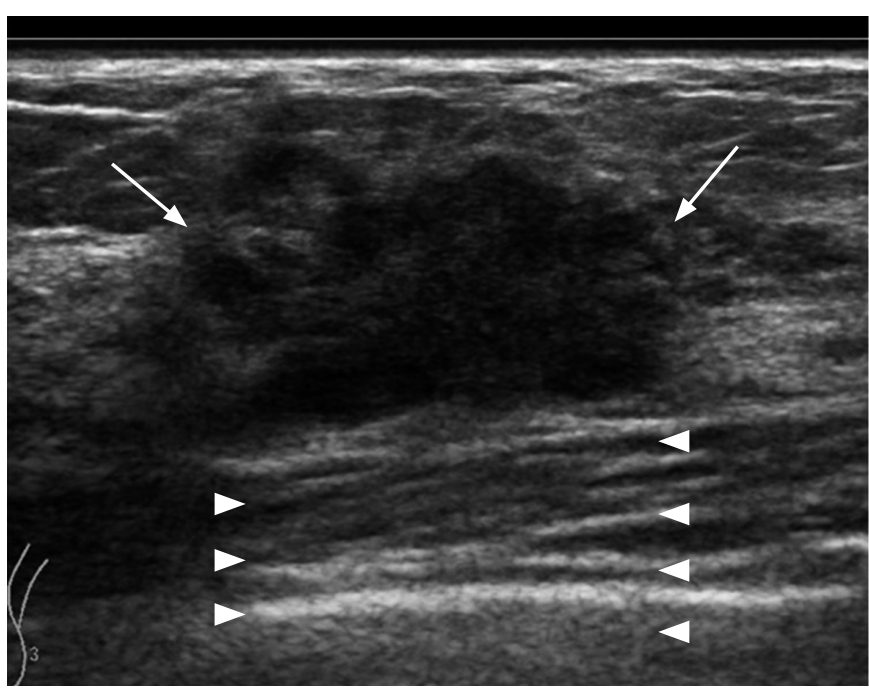

B

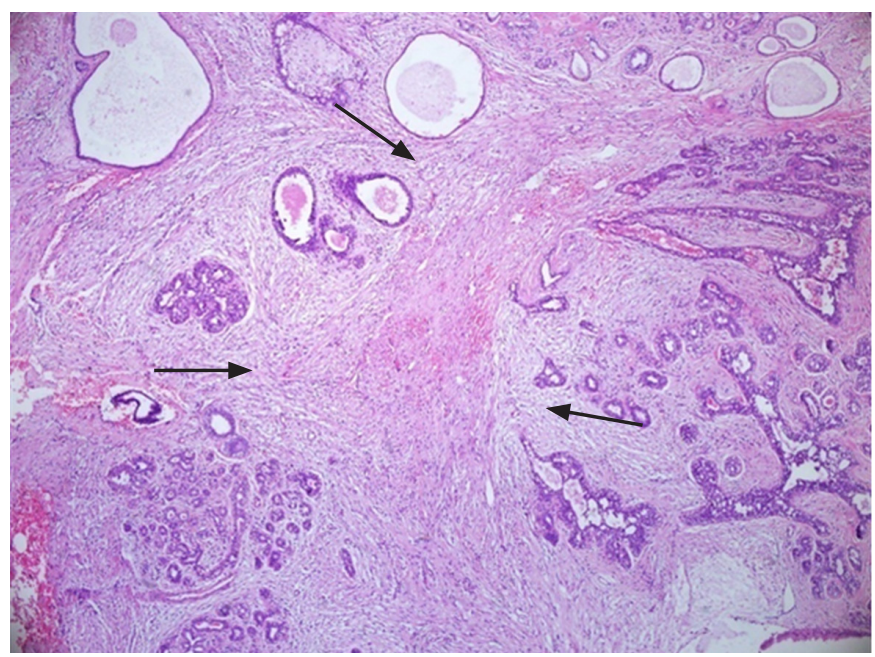

C 
diagnosis of complex sclerosing lesion based on ultrasonographyguided core needle biopsy that subsequently yield a diagnosis of ductal carcinoma in situ (DCIS) or invasive carcinoma after a surgical excision. After surgical excision, the rate of upgrade from complex sclerosing lesion to breast cancer (DCIS and invasive ductal carcinoma) was $28.6 \%$ ( 2 of 7 ).

\section{Discussion}

The association of the radial scar/complex sclerosing lesion with cancer is controversial $[10-12,14]$. Jacobs et al. [10] stated that radial scars are an independent risk factor for the development of breast cancer. In contrast, Berg et al. [11] stated that radial scars were not independent risk factors and that any increased risk was attributable to associated proliferative disease, such as atypical hyperplasia. Manfrin et al. [12] found that patients with coincident cancer generally had larger radial scar/complex sclerosing lesions. It has also been suggested that the size of the radial scar/complex sclerosing lesion may be important, with larger lesions being more likely to have malignant potential [12]. Therefore, many studies have suggested that a complex sclerosing lesion signifies the possibility of coincident cancer or precancerous lesions and that complete

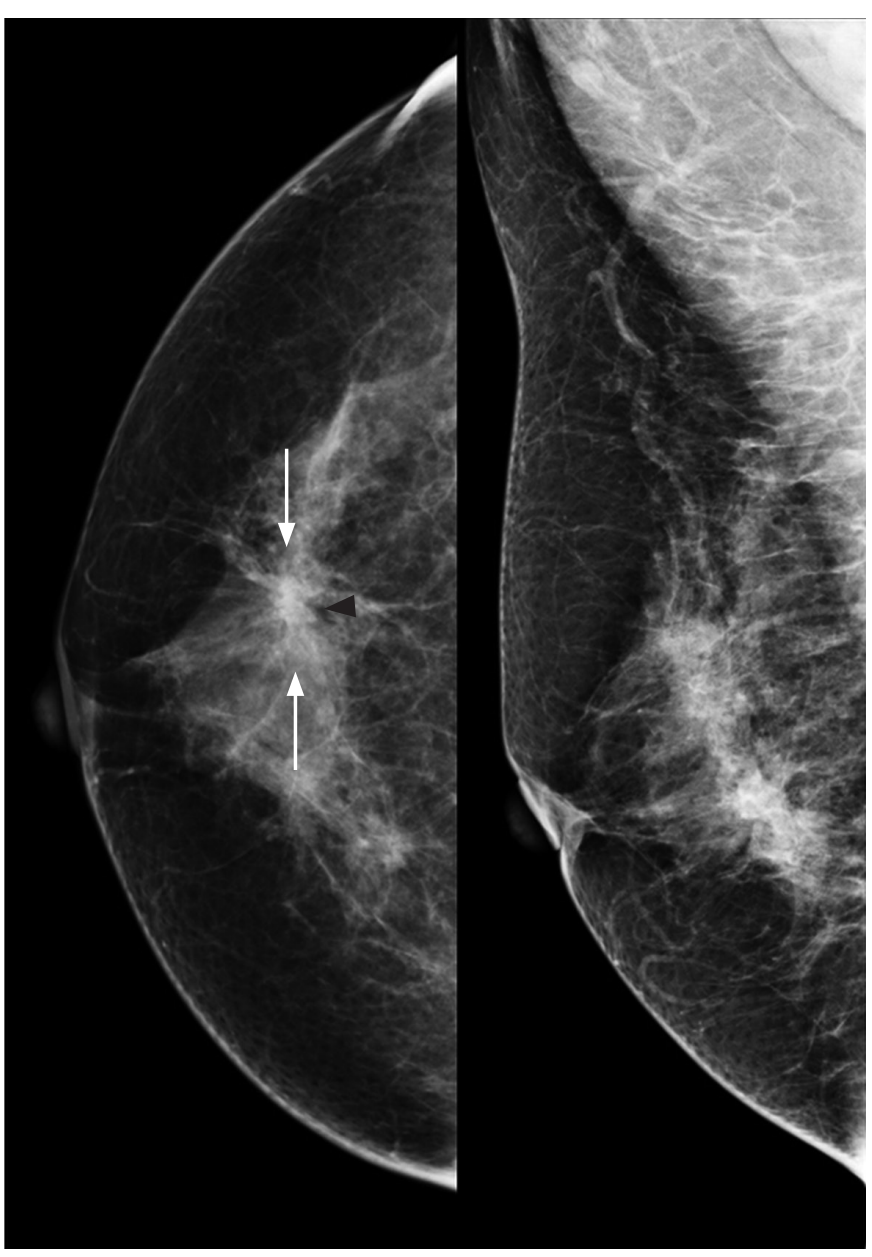

A

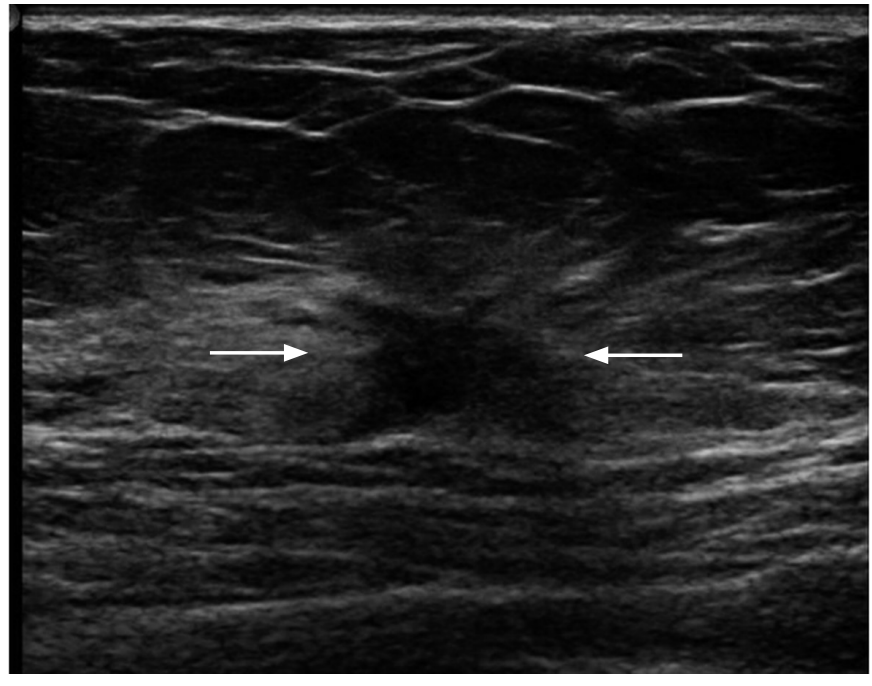

B

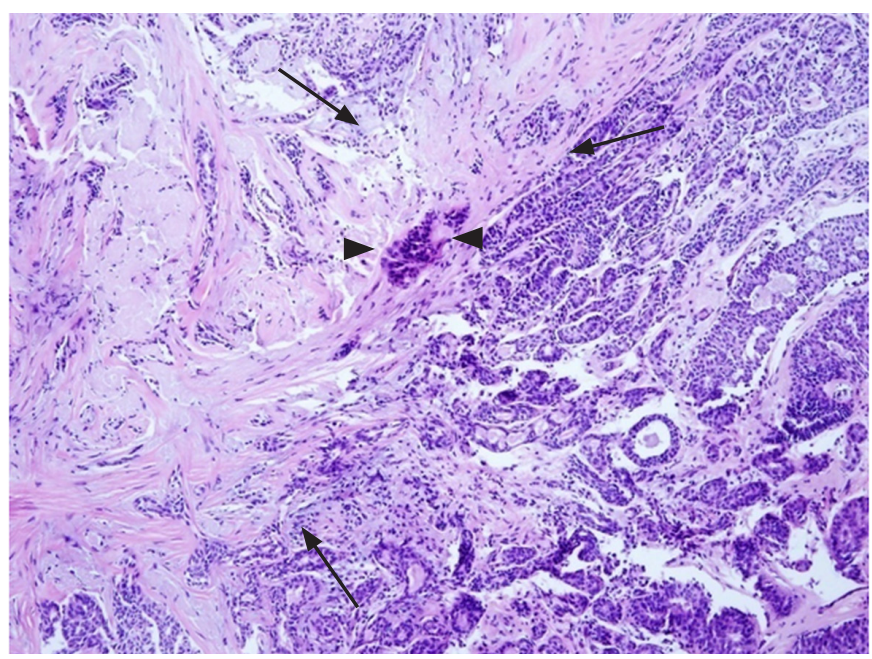

C

Fig. 3. A complex sclerosing lesion in a 51-year-old woman with no symptoms (patient 7).

A. A mammogram shows focal asymmetry (arrows) with a central radiolucent area (arrowhead). B. An ultrasonogram reveals an irregular, parallel, spiculated hypoechoic mass (arrows) with no posterior acoustic features. Upon BI-RADS assessment, it was classified into category 4C, with moderate suspicion of malignancy. C. A photomicrograph of a histologic specimen $(H \& E, \times 100)$ from excision reveals ductal carcinoma in situ (arrowheads) in the background of a complex sclerosing lesion (arrows). 
excision should be performed in older patients with larger radial scar/complex sclerosing lesions [2,9-11]. In our study, after surgical excision, two cases revealed malignancy, and one case revealed atypical ductal hyperplasia. Thus, the upgrade rate was $28.6 \%$. The current study showed the diagnosis of complex sclerosing lesion after imaging-guided biopsy is an indication for surgical excision to exclude the diagnosis of breast cancer.

On mammograms, complex sclerosing lesions exhibited a mass density in four patients that most commonly manifested as an obscured, oval, isodense mass. The lesions that appeared as isodense masses did not show a central radiolucent area or calcifications. The other patients exhibited focal asymmetry $(n=1)$ and architectural distortion $(n=2)$ with a central radiolucent area. Radial scars commonly present as asymmetric densities or architectural distortions with central translucent areas $[8,15]$. Long, radiating spicules against a background of radiolucent fat create a black, star-like appearance $[8,16]$. This appearance is in contrast to most of the complex sclerosing lesions, which appeared as masses that resembled breast cancer.

On ultrasonography, complex sclerosing lesions commonly manifest as irregular hypoechoic masses with spiculated margins (Table 3). Three cases showed posterior acoustic enhancement. Six cases were classified as category 4, which is characterized by suspicious findings. Radial scars commonly appear as hypoechoic lesions with irregular or spiculated margins and posterior acoustic shadowing [9]. However, in our study, the complex sclerosing lesions commonly appeared as irregular, hypoechoic masses with spiculated margins and posterior acoustic enhancement (Fig. 2B).

The limitation of this study is the small sample size. Because of the retrospective nature of the analysis and the selection bias for patients likely to be at risk of malignancy, large patient numbers will be required to improve the reliability in future studies. Furthermore, subsequent surgical excision was not performed for two lesions and one case was reviewed by a short-term follow-up imaging study (12 months) that can raise the possibility of breast cancer.

In conclusion, the present study showed a rate of upgrade to cancer of $28.6 \%$. A complex sclerosing lesion exhibited the etiology of a radial scar, but the imaging findings of a complex sclerosing lesion showed features distinct from those of a radial scar, such as their commonly appearing as a mass density on mammography and as an irregular, hypoechoic mass with a spiculated margin and posterior acoustic enhancement on ultrasonography. Therefore, all lesions that are pathologically proven to be complex sclerosing lesion by core needle biopsy should be excised.

ORCID: Joo Hwa Myong: http://orcid.org/0000-0002-8410-1102; Byung Gil Choi: http://orcid.org/0000-0002-2950-2069; Sung Hun Kim: http://orcid.org/0000-0003-
4478-9720; Bong Joo Kang: http://orcid.org/0000-0002-5991-6035; Ahwon Lee: http://orcid.org/0000-0002-2523-9531; Byung Joo Song: http://orcid.org/00000002-1461-5263

\section{Conflict of Interest}

No potential conflict of interest relevant to this article was reported.

\section{Acknowledgments}

This study was supported in part by the Research Fund of the Korean Society of Ultrasound in Medicine.

\section{References}

1. Sanders ME, Page DL, Simpson JF, Schuyler PA, Dale Plummer W, Dupont WD. Interdependence of radial scar and proliferative disease with respect to invasive breast carcinoma risk in patients with benign breast biopsies. Cancer 2006;106:1453-1461.

2. Fasih $T$, Jain M, Shrimankar J, Staunton M, Hubbard J, Griffith CD. All radial scars/complex sclerosing lesions seen on breast screening mammograms should be excised. Eur J Surg Oncol 2005;31:11251128.

3. Anderson TJ, Battersby S. Radial scars and complex sclerosing lesions. Histopathology 1994;24:296-297.

4. Kennedy M, Masterson AV, Kerin M, Flanagan F. Pathology and clinical relevance of radial scars: a review. J Clin Pathol 2003;56: 721-724.

5. Ellis IO, Pinder SE, Bobrow L, Buley ID, Coyne J, Going JJ, et al. Pathology reporting of breast disease: a joint document incorporating the third edition of the NHS breast screening programme's Guidelines for pathology reporting in breast cancer screening and the second edition of the Royal College of Pathologists' Minimum dataset for breast cancer histopathology [Internet]. Sheffield: NHS Cancer Screening Programmes jointly with The Royal College of Pathologists; 2005 [cited 2013 Nov 20]. Available from: http://www.cancerscreening.nhs.uk/breastscreen/ publications/nhsbsp58-low-resolution.pdf.

6. Sloane JP, Mayers MM. Carcinoma and atypical hyperplasia in radial scars and complex sclerosing lesions: importance of lesion size and patient age. Histopathology 1993;23:225-231.

7. Image interpretation session: 1996. Complex sclerosing lesion (radial scar) of the right breast masquerading as an occult breast carcinoma. Radiographics 1997;17:248-250.

8. Alleva DQ, Smetherman DH, Farr GH Jr, Cederbom GJ. Radial scar of the breast: radiologic-pathologic correlation in 22 cases. Radiographics 1999;19 Spec No:S27-S35.

9. Grunwald S, Heyer $H$, Kuhl A, Schwesinger G, Schimming A, Kohler G, et al. Radial scar/complex sclerosing lesion of the breast: value of ultrasound. Ultraschall Med 2007;28:206-211.

10. Jacobs TW, Byrne C, Colditz G, Connolly JL, Schnitt SJ. Radial scars 
in benign breast-biopsy specimens and the risk of breast cancer. $\mathrm{N}$ Engl J Med 1999;340:430-436.

11. Berg JC, Visscher DW, Vierkant RA, Pankratz VS, Maloney SD, Lewis JT, et al. Breast cancer risk in women with radial scars in benign breast biopsies. Breast Cancer Res Treat 2008;108:167-174.

12. Manfrin E, Remo A, Falsirollo F, Reghellin D, Bonetti F. Risk of neoplastic transformation in asymptomatic radial scar. Analysis of 117 cases. Breast Cancer Res Treat 2008;107:371-377.

13. D'Orsi CJ, Mendelson, EB, Ikeda DM, eds. Breast Imaging Reporting and Data System: ACR BI-RADS-Breast Imaging Atlas. Reston, VA: American College of Radiology, 2003.
14. Bunting DM, Steel JR, Holgate CS, Watkins RM. Long term follow-up and risk of breast cancer after a radial scar or complex sclerosing lesion has been identified in a benign open breast biopsy. Eur J Surg Oncol 2011;37:709-713.

15. Inoue $S$, Inoue $M$, Kawasaki T, Takahashi $H$, Inoue A, Maruyama $\mathrm{T}$, et al. Six cases showing radial scar/complex sclerosing lesions of the breast detected by breast cancer screening. Breast Cancer 2008;15:247-251.

16. Pediconi F, Occhiato R, Venditti F, Fraioli F, Napoli A, Votta V, et al. Radial scars of the breast: contrast-enhanced magnetic resonance mammography appearance. Breast J 2005;11:23-28. 